PREHOSPITAL CARE

\title{
"Reforming Emergency Care": the ambulance impact. A personal view
}

\section{Robertson-Steel}

Emerg Med J 2004;21:207-211. doi: 10.1136/emi.2002.001669

\begin{abstract}
"Reforming Emergency Care" has huge implications for ambulance services to develop their future role and the opportunity to improve patient care. This article aims to stimulate debate on the effect on ambulance services in the United Kingdom.
\end{abstract}

Correspondence to: Dr I Robertson-Steel, West Midlands Ambulance Service NHS Trust, Millennium Point, Waterfront Business Park, Waterfront Way, Brierley Hill, DY5 ILX, UK; iain.robertson-steel@ wmas.nhs.uk

Accepted for publication 11 May 2003
$\mathrm{R}$ forming Emergency Care $^{12}$ was launched on Thursday 25 October 2001 by the Secretary of State for Health. At the time of writing, 18 months has passed, demand continues to rise, and the National Health Service remains under pressure. The launch of the NHS Plan ${ }^{3}$ in 1997 envisaged new ways of integrating health and social care; little change has occurred.

In the year 2001-2002, 4.7 million emergency calls were made and the number of incidents attended continued to rise, reaching 3.8 million.

"The New NHS: Modern-Dependable" outlined the need for a re-appraisal of roles and new ways of working. ${ }^{4}$

The ambulance services faced a rise in emergency patient journeys to 3.1 million.

Across the country, accident and emergency departments remain under increasing and unremitting pressure, with ambulance services suffering increasing delays at turn around, when patients are brought to accident and emergency.

The demands on NHS Direct have increased and general practice awaits the delivery of a new contract, which has potentially significant effects on out of hours services.

One year on, few members of the public or the health service would recognise radical change following Reforming Emergency Care's publication. However, a great deal of work continues to be done across the United Kingdom to respond to the challenges of increasing demand. Ambulance services have made significant progress in meeting government targets, but evidence of improvement in patient outcome is lacking. Nicholl et al reviewed the future possible direction in $2000 .^{5}$

Reforming Emergency Care is a long term plan; one year on, it is now appropriate to consider Reforming Emergency Care and what impact it will have on ambulance services. The underlying objectives of Reforming Emergency Care could be categorised as follows.

- Firstly, to treat patients on the basis of clinical need, treating the sickest first.

- Secondly, to deliver definitive care to patients in the most appropriate facility in minimum time, in the most appropriate facility.
- Thirdly, to maximise the use of resources available to the greatest patient and healthcare benefit.

Reforming Emergency Care identified the need to change the prioritisation system. It specified a new call prioritisation system for the NHS, which would quickly identify conditions or circumstances that have potential to be life threatening. Where need is not time critical, patients would be assessed promptly and effectively and directed to the most appropriate service. It was perceived that this would entail patients not going to hospital by ambulance, but by a range of alternative paths to other services instead. Implicit in this policy is the need to ensure that new portals are opened up in partnership.

New roles for ambulance service and paramedic staff were envisaged, to strike a balance between emergency ambulance response and patient transport services.

One year on, no single prioritisation system applicable across the NHS has been identified or developed. The United Kingdom Ambulance Services are using AMPDS (Advanced Medical Priority Dispatching System) and CBD (Criteria Based Dispatching) and continue to dispatch ambulances on the basis of AMPDS category.

Recent development work at West Midlands Ambulance Service ${ }^{6}$ has been the emergence of an emergency nurse advisor project, which takes the category alpha calls from AMPDS, arising from a domestic address, assesses them, and reprioritises them. This project has shown some promise, but has been limited in its ability to improve health care by the shortage of alternative transport systems and by the inability of other healthcare partners to take patients by alternative referral routes. The project has also been limited by constraints relating to target times.

NHS Direct has made progress with the piloting of the Primary Patient Prioritisation System, which has been able to identify patients with life threatening problems who ring NHS Direct, and safely and satisfactorily transfer them to the ambulance service. Further work is taking place to develop streaming systems after prioritisation to direct the patient to the most appropriate health service resource, and to assist in capacity management.

An editorial on this topic has been published in the journal (2004;21:4). 


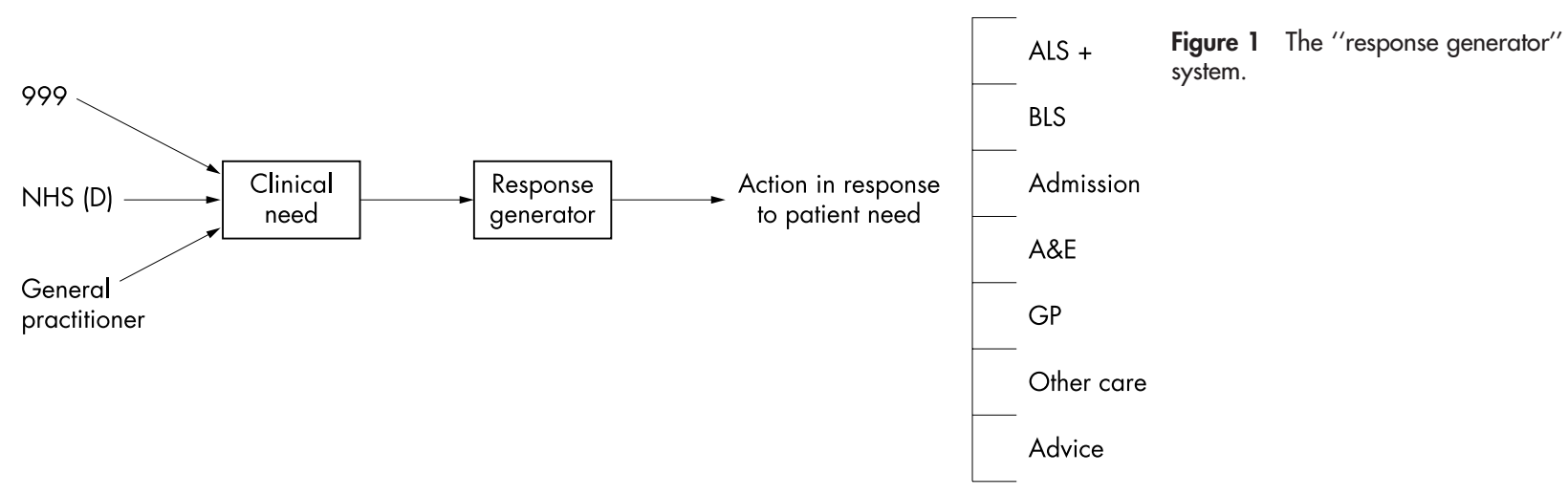

Out of hours general practitioner services are not formally triaging referrals to hospital by way of the ambulance service, and in an emergency, general practitioners continue to dial 999. To operate a single queue system for seriously ill patients, a new approach is needed to ensure that GP referrals receive an appropriately rapid response.

For the foreseeable future, it is unlikely that we will see the emergence of a single NHS prioritisation system.

The work to date, from the NHS Direct Primary Prioritisation Project and WMAS ENA project, suggests that AMPDS and CBD (Criterion Based Dispatching) can work in parallel with NHS Direct systems to identify immediate life threatening risks.

Further work needs to be carried out to develop the ability of ambulance services to transfer calls to NHS Direct. The DoH, in the Emergency Care Toolkit, ${ }^{7}$ identifies NHS Direct as taking the low priority 999 calls. $^{7}$

To prioritise all patients entering the system on the basis of clinical need, general practice will need to develop a system where the GP can prioritise calls as "immediate" (immediately life threatening), "urgent", and "sequential".

In the future, it is probable that there will not be a single NHS prioritisation system, but it is more likely that the existing systems will be developed to refer cases into a "response generator", which will deliver the best available local response on the basis of clinical need (fig l).

For a response generator to work, a clearer understanding of all parties and the public will be required as to what constitutes a life threatening emergency and what cases can be safely deferred to alternative portals of care, such as walk in centres, general practice, and minor injury units.

As an example, cases categorised as "delta" or "Charlie" on AMPDS would receive an immediate paramedic response, whether they arose from a 999 call, NHS Direct, or a GP.

Cases below category $\mathrm{C}$ on AMPDS would be subject to further triage using the CAS (Clinical Advisory System) as used by NHS Direct, to generate the priority and identify the best available local resource.

The UK NHS Direct experience to date suggests that patients can be directed safely into lower priority categories, the problem being arranging routes of access for patients who are not prioritised as requiring an A\&E department by the conventional ambulance route. Further work and outcome studies need to be undertaken.

For ambulance services to focus on emergency care and to improve the delivery of care to those who are at immediate risk, the only option available is to focus specialist resources on those at immediate life threatening risk and refer patients who do not require a paramedic response to alternative resources. This will maximise the use of staff, vehicles, and expertise.
If patients are prioritised, the key to the future is having partnerships with other healthcare providers.

If NHS Direct and ambulance services identify patients as requiring transport to a walk in centre, a minor injuries unit, or a response from a GP by home visit, or transportation to their surgery, this should be achievable. Partnerships with local emergency care networks will be required to develop the best available local resource to meet patient need.

In the future, it would be expected that no cases of minor illness will be transported to an A\&E department, but they would be taken to GP out of hours facilities instead.

For the system to function, alternative points of access to health care must be available and clearly understood by all partners.

If one million 999 cases per annum could be diverted to alternative, appropriate care, this would significantly improve the delivery of care to the critically ill and injured, by freeing up resources to meet demand.

It is entirely inappropriate that at the present time, patients who dial 999 with minor problems should take priority over sicker patients who have been seen by a general practitioner.

In the future, it will be necessary, using a response generator, to effectively develop a single queue system for all patients requiring emergency care.

For the future, therefore, work needs to continue on a national response generator, to integrate AMPDS, CBD, NHS Direct, Primary Prioritisation, and GP requests.

In the future, for emergency nurse advisor projects to develop and flourish, alternative sources of transport should be available and alternative portals of access to health care at

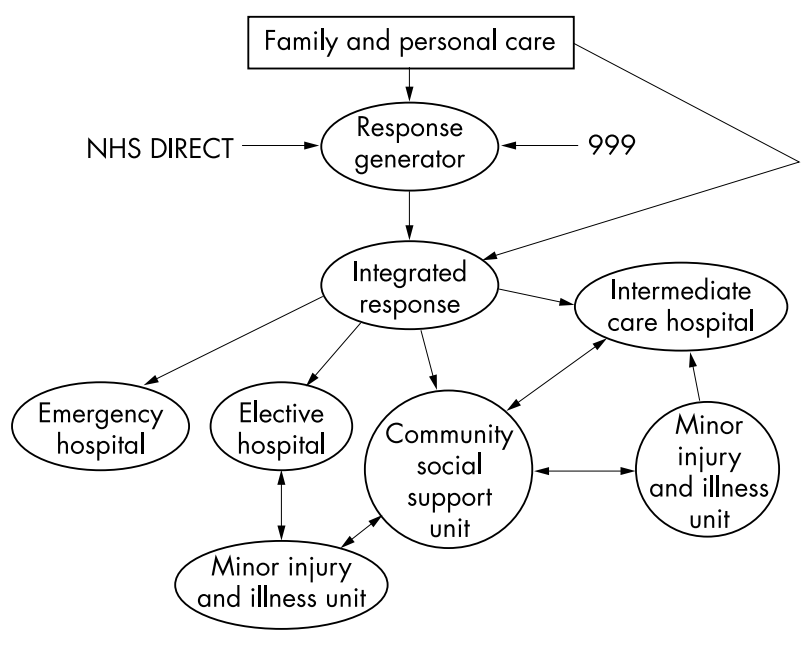

Figure 2 Local care group. 
walk in centres, GP out of hours centres, minor injuries units, and, possibly, A\&E departments will be required. Whether emergency nurse advisors are necessary in ambulance control centres or whether work can be passed directly to NHS Direct, needs to be clarified by further pilot work.

Local emergency care networks will have to agree standards of access for appropriate patients to ensure that care is delivered on the basis of clinical need.

It must be emphasised that inherent in all prioritisation systems, there are limitations to sensitivity and specificity. It must be expected that any new system must accurately classify patients on the basis of need, in the vast majority of cases. It should not be seen as the failure of the system if patients are subsequently re-prioritised or subjected, on occasion, to secondary transfer.

In the future it is essential for UK ambulance services to know what services are provided by each healthcare facility. It may be necessary, in the future, to focus on the development of major illness and injury centres, with A\&E departments staffed by consultants 24 hours a day, with prompt access to computed tomography and magnetic resonance scanning. Clearly, with the existing levels of staff and resources, this may need reconfiguration of A\&E departments to change the model from the "9 to 5" A\&E department with an out of hours service, to a model where 24 hour major illness and injury centres are available, supported by satellite minor injury centres.

In the future, it may be necessary to reconfigure entire healthcare systems into emergency centres and emergency hospitals, elective hospitals with attached minor injury units, and GP out of hours centres for minor illness (fig 2).

If these centres were appropriately provided, the ambulance service task would be to deliver appropriate patients, using the correct level of transport where required, to the correct unit. In the short term, maximum efficiency of ambulance services can be achieved by being able to defer and divert patients to alternative sources of care, and also by reducing ambulance service delays in A\&E departments.

The key is to be able to identify those patients who should go to $A \& E$, and divert to the appropriate level of health care those who should not.

By 2004/2005, NHS Direct will be handling all GP out of hours emergency calls. NHS Direct will have a Primary Prioritisation system that will effectively identify patients at immediate life threatening risk. This system will be rolled out across the country, over the next year, and a streaming component is under development.

Further development work is taking place on NHS Direct Clinical Advisory System (NHS Direct CAS) and in the future the system needs to be simplified. Outcomes in NHS Direct CAS need to be sorted into Urgent, in other words those patients who need to see a healthcare professional with further examination and a clinical opinion at the earliest possible opportunity, and deferred for those patients who can wait until normal hours NHS facilities are available.

To make Reforming Emergency Care effective, clear local and national agreement must be reached on cases that can appropriately be sent to A\&E, cases that are appropriate for a minor injuries unit or walk in centre, and cases that must go and see a GP.

In the future, the use of $A \& E$ as a default option will no longer be acceptable.

General practice at the present time is under pressure, the new General Practitioner Contract, if accepted, offers GPs the option of opting out of out of hours care. This responsibility will then remain with the Primary Care Trusts. There has been much recent talk of paramedics taking on some of the roles of GPs. At the present time, the existing cadre of paramedics are fully committed delivering emergency care, and it is unlikely that reconfiguration of services will free up paramedic time to carry out GP work. It is possible, however, to increase the number of paramedics and give them further training and new skills. I will discuss this point later.

Reforming Emergency Care encourages GPs to seek new ways of working. From the ambulance service perspective, the need for GPs to participate in triage is vital. GPs will need advice and training on what patients constitute an immediate case, and these patients arising from general practice, should be treated with the same priority as they would have been given had they accessed through AMPDS on the 999 system. The principle has already been established for myocardial infarction patients.

GPs need to address the issues of being able to classify patients into an agreed "urgent" category, those patients who do require an ambulance to remove them to hospital, and those patients who require transport purely for admission.

General practice also needs to reconfigure the way in which it works. It is inappropriate for ambulance services to experience heavy demand around mid-day, when GPs start the home visits, seeing their sicker patients when they have already carried out a surgery.

For ambulance services and A\&E departments to work effectively, if GPs are able to start their visits earlier in the day, this would have benefits to both the ambulance service and the A\&E departments and admissions units.

\section{HOW CAN AMBULANCE SERVICES “WORK SMARTER"}

Ambulance services should continue to develop and refine prioritisation systems, and develop their cross links with NHS Direct. In the future, ambulance services will have to develop alternative transport systems, perhaps using cars for basic patient transport for the mobile, and an assisted taxi servicetype vehicle for those who require help and support but not critical care facilities.

This will result in reconfiguration of ambulance service vehicles and responses.

In large urban areas, it may be necessary to have medically qualified controllers assisting in prioritisation and in the management of complex cases. This model works well in many parts of Europe. This model will be greatly assisted by simple telemedicine links, allowing the care of critically ill patients to be planned from the time of call and the arrival of the ambulance crew directly to the nearest appropriate resuscitation facility.

\section{WHAT OF THE FUTURE OF TRAINING FOR AMBULANCE SERVICE HEALTHCARE PROFESSIONALS?}

In the future, there will be a continuing need for clinicians who are capable of dealing with resuscitation and the management of major adult medical emergencies and major adult trauma. These clinicians will also require skills in the management of the critically ill and injured child. It is unrealistic to expect all ambulances to carry advanced paramedics; the expectation clearly is that where they are needed, they should be sent. This will be dependent on improved prioritisation and triage systems and, for the foreseeable future, there will remain a need to have paramedics capable of delivering essential immediate care to the critically ill and injured, and rapidly treating and transporting them to the nearest appropriate facility. The concept of minimum time to definitive treatment suggests that paramedics should be able to carry out rapid assessment and institute advanced treatment to the Joint Royal College Ambulance Liaison Committee version 2 guidelines 
standard, ${ }^{8}$ where appropriate. In the future, it is likely that these paramedics will be trained to diploma and subsequently degree level.

\section{WHAT EXTRA ROLE COULD THESE PERSONNEL TAKE ON?}

A Health Service of all the Talents ${ }^{9}$ (DoH 2000a) emphasised a number of issues, including team working across professional and organisational boundaries. The University of Central Lancashire and Birmingham and Black Country Workforce Development Confederation project summarised the challenges ${ }^{10}$

This group of staff will have to be trained to a much higher degree in the ability to assess a patient. This will benefit the management of the critically ill and injured, but will also allow them to assess patients and make decisions to manage those patients in new ways. If a paramedic is able to carry out an accurate assessment of a patient, it may be possible for the paramedic to treat the patient and leave the patient at home or, more likely, to examine and assess the patient and then decide on where the patient needs to go for further treatment, without an interim staging in A\&E. Hospital medical staff will, in the future, need to be prepared to accept direct referrals from ambulance service staff.

The concept of assessing and referring will allow paramedics, in the future, to take patients directly to a medical admissions unit, a surgical admissions unit, or a GP's surgery. This will have very beneficial effects on A\&E departments and will save significantly on patient waiting time by avoiding the unnecessary step of A\&E. With training, paramedics should accurately be able to diagnose strokes, acute abdomens, and patients with life threatening medical problems who will require admission. By allowing paramedics to give essential treatment and then take the patient directly to the most appropriate unit, this will significantly improve the efficiency of the healthcare system and reduce unnecessary re-triage, re-clerking, and delays in A\&E.

This group of clinicians will also be able to assess patients and, where appropriate, take them directly to general practice, where the GP will be able to arrange further investigations and/or provide treatment which the paramedics are not currently able to do.

If a patient is visited by this type of clinician, it may be possible to arrange admission to hospital using patient transport services, rather than a front line ambulance. Many of these clinicians would be capable of operating as independent practitioners, carrying out assessment of patients and then arranging the most appropriate health care for them, and the most appropriate transportation.

This group of practitioners, by operating solo, would be, in addition, an effective cadre of first responders who would be available to assist ambulance crews and to meet the eight minute response target.

It is not appropriate to consider the paramedics as an alternative source of nursing staff for A\&E departments. The current satisfactory recruitment of paramedics and technicians is for their existing role. This is not a hospital based role and it is unlikely, in the present model, that paramedic staff would wish to work in an A\&E department, providing nursing care.

In the longer term, as the skill base and the training of paramedics is extended, it is possible that paramedics may be able to be attached to general practices and minor injuries units, but this will require a considerable change in the prescribing capabilities of paramedics, and further training.

If emergency services are able to develop effective prioritisation systems, "urgent" patients who do not require the services of paramedics and advanced clinicians will be able to be transported by emergency medical technicians with an extended role.

Reforming Emergency Care places heavy emphasis on clinical decision support systems, electronic patient records, and IT systems. It is vital for the future that the development of such systems is carried out on a planned and integrated basis. All clinicians, whether they are paramedics, technicians, NHS Direct nurses, will require access to the patient's records and clinical decision support systems. A national standard for ambulance service e-PRF and clinical decision support, and access to patient records is urgently required.

National standards for ambulance services, A\&E departments, acute trusts and primary care, need to be set as a matter of urgency, to prevent divergent development of local systems.

\section{WHAT OF THE FUTURE?}

The NHS is having to tackle the issues of staff shortages, increasing public expectation, and an aging population. The aging population will generate an increasing demand for the management of chronic diseases.

Partnership is the key to the future. All partners in health care must operate using the same prioritisation systems. All partners must understand the capabilities and skills of other partners, and each must fully understand the role and responsibilities of all participants.

The future will see out of hours access to health care, either by the 999 route, or through NHS Direct. Public and all healthcare professionals must clearly understand how to access health care, and must operate on the driving principles of treating the sickest first, minimum time to definitive treatment, and maximising the use of available resources.

Reforming Emergency Care will generate fundamental changes in the way health care is delivered. Perhaps the future lies in the development of Integrated Emergency Care Trusts, with ambulance services, NHS Direct, and out of hours GP and clinical services working together with social care.

The existing model, with 999 services, NHS Direct, and general practice, operating relatively independently of each other, without common memoranda of understanding on referral pathways, is no longer sustainable.

The emergency versus elective targets debate will need to be resolved. It is unlikely, in the future, that district general hospitals, providing open access A\&E departments, will continue to exist, or that A\&E will be the only point of access for the ambulance service.

Partnership for the future is the key. Ambulance services and their NHS Direct partners have the skills to coordinate and integrate out of hours care. They currently cover very large areas and multiple hospitals.

With the emergence of emergency care networks, it may be appropriate to consider these as care networks, providing the pathways for the delivery of appropriate patient care 168 hours a week. In the future it may be necessary to reconfigure hospitals and their front door A\&E services to create emergency care hospitals with no walk in access, elective hospitals with attached minor injuries, minor illness, and GP out of hours centres and intermediate care hospitals (see fig 2).

If all healthcare professionals were employed by the same local care network, perhaps based on a population of one million, it would be possible to improve care by working together rather than in competition.

If all of the healthcare professionals were employed by the "Local Care Group", with the ambulance Service, NHS Direct, primary care trusts and hospitals working together, artificial targets and competition would be removed. Performance of the healthcare system could then be measured on its ability 
to deliver care to its catchment population, without artificial and perverse incentives. The success of a healthcare system would be based on the ability of its local care network to deliver integrated emergency care, and to provide care for the critically ill and injured, those with a need for hospital admission, and those who can be managed by general practice and outpatient care.

Reforming Emergency Care has huge implications for ambulance services. Ambulance services will move away from being transport systems to being integrated systems for the delivery of care by a wide range of professionals with access, on an agreed basis, to many portals of the health services. Ambulance services, working together with NHS Direct, are likely to become the integrators of health care and the custodians of the electronic patient record.

By working in partnership with the acute trusts and primary care, integrated care trusts will prioritise patients' needs, direct them to the most appropriate resources and arrange for their transportation, whether they be an emergency requiring resuscitation or referral to a tertiary unit directly, or simply arrange for the delivery of the patient to general practice or the GP, or indeed another skilled clinician to make an assessment.

Reforming Emergency Care is a challenge and an opportunity for ambulance services that should not be missed. This is not an opportunity for ambulance services to become the controllers of the system, but to become the integrators of the system in partnership.
The embryonic emergency care networks are the first stage in the development of integrated care for the community. By delivering care on the basis of clinical need and directing those whose needs are less urgent to the appropriate level of care, the overall benefit to the patient can be greatly improved.

Reforming Emergency Care truly empowers ambulance services to develop their future destiny as the integration and emergency arm of healthcare-an opportunity to improve patient care that must not be missed.

\section{REFERENCES}

1 Department of Health. Reforming emergency care. Norwich: The Stationery Office, 2001

2 Department of Health. Reforming emergency care: first steps to a new appraisal. London: DoH, 2002

3 Department of Health. The NHS plan. London: DoH, 1997.

4 Department of Health. The new NHS: modern-dependable. Command 3807. London: DoH, 1997b.

5 Nicholl J, Turner J, Martin D. The future of ambulance services in the United Kingdom. Sheffield: Medical Care Research Unit, Scharr/ASA, 2000.

6 The ENA Project. West Midlands Ambulance Service NHS Trust. Modernisation Agency web site, 2002 (http://www.modern.nhs.uk).

7 Department of Health. Emergency care toolkit, 2003. http:// www.doh.gov.uk/emergencycare/aetargetchecklist.htm.

8 JRCALC. Pre-hospital guidelines for use in UK ambulance services (v. 2.2) London: Joint Royal Colleges Ambulance Liaison Committee and Ambulance Service Association (ASA), 2002.

9 Department of Health. A health service of all the talents. London: DoH, 2000

10 University of Central Lancashire, Faculty of Health. Establishing a diploma level education programme for ambulance service paramedics in the West Midlands, 2002 (http://www.wmas.nhs.uk/publications). 